\title{
Impact of nudging strategies on consumer food choice intentions and behaviours in leisure centres
}

\author{
P.A. Ashworth, C. Morris, S. Giove and J.R. Paxman \\ Food and Nutrition Subject Group, Sheffield Business School, Sheffield Hallam University, Sheffield, S1 1WB, UK
}

The inherent leptogenic (lean promoting) physical activity environment in leisure centres, offers a platform for the communication of leptogenic food behaviours ${ }^{(1)}$. Nudging theory posits that the architecture of an environment, such as the design or availability of information, can influence consumer choice ${ }^{(2)}$. Nutritional information can also enable informed decisions ${ }^{(3)}$. The Theory of Planned Behaviour (TPB) hypothesises that attitudes, social norms, and perceived behavioural control (PBC) inform consumer intention, and collectively predict behaviour ${ }^{(4)}$. The aim of this study was to determine the impact of a nudging strategy, in the form of the provision of calorie information, on personal intention and food choice behaviour in leisure centre cafés.

The experiment comprised of a week-long pre-intervention stage (week 1) and a week-long intervention stage (week 2). During week 2 , Calorie information was introduced onto the menu in an experimental centre $(n 1)$, but not in the control centre $(n 1)$. A questionnaire informed by the $\mathrm{TPB}^{(4)}$, adapted to include concern, motives, confidence, and actual food choice, was distributed throughout the study to café users ( $n$ 323) in both centres. The mean scores for each construct, and the calorie content of food choices between the two weeks, in each of the centres, were compared using independent samples t-tests.

Fig 1 shows that the nudging strategy significantly increased confidence $(\mathrm{p}=.022)$ and $\mathrm{PBC}(\mathrm{p}=.001)$ with regards to leptogenic food choices. This was not observed in the control centre (Fig 2). A downward trend in the average Calories consumed was observed for snack items $(p=\cdot 144)$, and lunches $(p=.140)$ consumed in the experimental centre (Fig 1). Average Calories consumed were 177 Calories lower for lunch, and 110 Calories lower for snacks during week 2, compared to week 1. A less pronounced downward trend was observed for snacks $(p=.505)$, but not for lunches $(p=.992)$, in the control centre (Fig 2$)$.

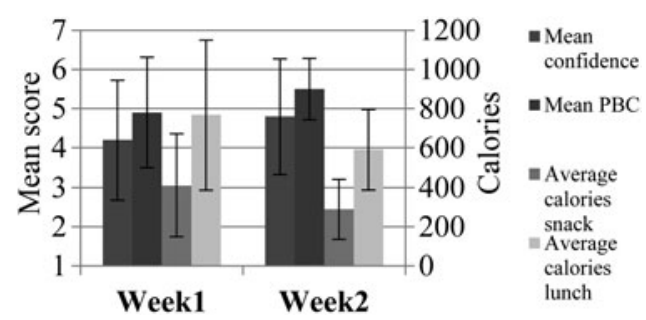

Fig. 1. Experimental Centre.

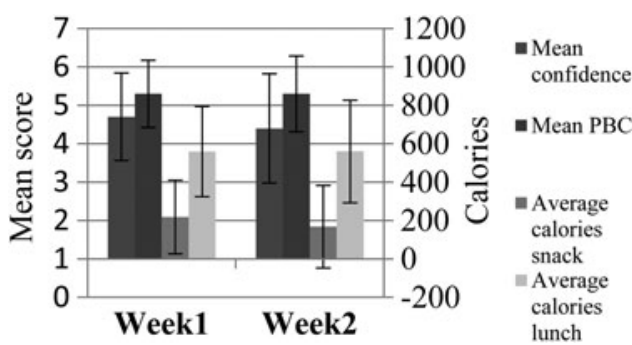

Fig. 2. Control Centre.

Calorie information has the potential to change consumer interaction with the food environment in leisure centres, and increase leptogenic food behaviours. Further stages to this research will include an assessment of the goodness of fit of the adapted TPB model.

1. Carter M, Signal L, Edwards R et al. (2013) BMC Public Health 13, 1-7.

2. Olstad DL, Goonewardene LA, McCargar LJ et al. (2014) Int J Behav Nutr Phys Act 11, 1-14.

3. Hodgkins CE, Raats MM, Fife-Schaw C et al. (2015) Br J Nutr 113, 1652-1663.

4. Ajzen I (1991) Organ Behav Hum Decis Process 50, 179-211. 